\title{
Exceso de peso, hábitos alimentarios y de actividad física de niños y niñas residentes en áreas urbanas y periféricas de la ciudad de La Plata
}

\section{María Florencia Cesani ${ }^{1}$ y Evelia Edith Oyhenart ${ }^{2(\dagger)}$}

\author{
1 Laboratorio de Investigaciones en Ontogenia y Adaptación (LINOA). Facultad de Ciencias Naturales y \\ Museo (FCNyM), Universidad Nacional de La Plata (UNLP); Consejo Nacional de Investigaciones Científi- \\ cas y Técnicas (CONICET). La Plata, Argentina. \\ (iD) https://orcid.org/oooo-0003-1372-9231 \\ Correo electrónico: mfcesani@fcnym.unlp.edu.ar \\ 2 Laboratorio de Investigaciones en Ontogenia y Adaptación (LINOA). Facultad de Ciencias Naturales y \\ Museo (FCNyM), Universidad Nacional de La Plata (UNLP); Consejo Nacional de Investigaciones Científi- \\ cas y Técnicas (CONICET). La Plata, Argentina. \\ (iD) https://orcid.org/oooo-0003-1331-7368 \\ Correo electrónico: oyhenart@fcnym.unlp.edu.ar
}

Recibido:

4 de mayo de 2020

Aceptado:

4 de junio de 2021

doi: $10.34096 /$ runa.v42i2.8361

\section{Resumen}

La urbanización creciente y acelerada promueve patrones de vida sedentarios y de alimentación poco saludable que incrementan el sobrepeso y la obesidad infantil. Desde una perspectiva antropológica, el objetivo del trabajo fue conocer las prevalencias de exceso de peso y los hábitos alimentarios y de actividad física de niños y niñas residentes en el casco urbano y en la periferia de la ciudad de La Plata. Participaron del estudio escolares de ambos sexos con edades comprendidas entre los 6 y los 13 años. Aproximadamente el $44 \%$ presentó exceso de peso. Los hábitos alimentarios y de actividad física fueron relativamente saludables y similares en los niños con estado nutricional adecuado y con exceso de peso. No obstante, se reconocieron diferencias, según el lugar de residencia, vinculadas con prácticas características de entornos obesogénicos, tales como el uso del televisor durante las comidas, la omisión del desayuno, el consumo de alimentos hipercalóricos y el picoteo entre las comidas principales.

Excess weight, feeding and physical activity habits of children residents in urban and peripheral areas of La Plata city

\begin{abstract}
The accelerated urban growth promotes sedentary and unhealth life habits that increase childhood overweight and obesity. The objective of this work was to know from an anthropological perspective, the prevalence of excess weight
\end{abstract}

\section{Palabras clave}

Sobrepeso; Obesidad; Alimentación; Actividad Física; Estudiantes

Key words

Overweight; Obesity; Feeding habits; Physical activity; Students 
and eating and physical activities habits of children residents in the Urban District and the periphery of the city of La Plata. Schoolchildren aged 6 to 13 of both sexes participated in the study. Approximately $44 \%$ presented excess weight. Eating and physical activity habits were relatively healthy and similar in children with adequate nutritional status and with excess weight. However, differences were recognized, depending on the children's place of residence, linked to practices characteristic of obesogenic environments, such as the use of the television during meals, the omission of breakfast, the consumption of foods rich in carbohydrates and the snacks between principal meals.

\section{Sobrepeso e obesidade, hábitos alimentares e atividade física das crianças residentes na zona urbana e periferia da cidade de La Plata}

\section{Resumo}

Palavras-chave

Sobrepeso; Obesidade; Hábitos alimentares; Atividade física; Estudantes
A urbanização crescente e acelerada promove padrões de vida sedentários e hábitos alimentares pouco saudáveis que aumentam o excesso de peso infantil. A partir de uma perspectiva antropológica, o objectivo do trabalho foi conhecer a prevalência do excesso de peso e os hábitos alimentares e de atividade física das crianças que vivem na zona urbana e na periferia da cidade de La Plata. A amostra foi constuída por crianças de ambos os sexos (6-13 anos de idade), dos quais $44 \%$ tinham excesso de peso. Os hábitos alimentares e de atividade física foram relativamente saudáveis e semelhantes em crianças com estatuto nutricional adequado e com excesso de peso. No entanto, foram observadas diferenças, dependendo da zona de residência, ligadas a práticas características dos ambientes obesogênico, tais como não tomar café da manhã, comer enquanto se vê televisão, consumir alimentos ricos em hidratos de carbono e comer snacks.

A “Chichi” Oyhenart, amiga y colega, por sus valiosos aportes a la antropología biológica, sus diarias enseñanzas y su apoyo incondicional para la realización de este trabajo.

La alimentación entendida como hecho social total permite, a partir de su desnaturalización, acceder a las dinámicas sociales que la subyacen (Carrasco Henríquez, 2007). Esta concepción admite que la alimentación es un hecho complejo y que es pasible de descomponerla en las dimensiones biológica, económica, política, cultural y simbólica (Díaz Córdova, 2016). Debido a ello, varios autores han señalado la necesidad de efectuar estudios interdisciplinarios que permitan comprender las distintas dimensiones y los aspectos que se conjugan en dicho proceso (Aguirre, 2004; Franco Patiño, 2010). Es así que, desde la interdisciplinariedad y la complementariedad de los métodos, las ciencias naturales y las ciencias sociales han comenzado a articular esfuerzos para estudiar las manifestaciones alimentarias y lograr el conocimiento integral de dicho fenómeno (Franco Patiño, 2010).

En tanto reflejo de la alimentación, uno de los posibles ejes de análisis es el estudio del estado nutricional del individuo y de las poblaciones, que se define como la condición corporal que resulta del balance entre la incorporación de nutrientes (a partir de la ingesta) y su utilización por parte del organismo (Pellegrini y Battistini, 2015). Cuando existe un desequilibrio entre la ingesta 
y el gasto de energía hablamos de malnutrición, término que refiere tanto a la malnutrición por déficit (desnutrición) como por exceso (sobrepeso y obesidad) (Organización Mundial de la Salud, 2020).

A nivel global, un reciente estudio realizado por el Fondo de las Naciones Unidas para la Infancia (UNICEF, 2019) daba cuenta de que la malnutrición, lejos de ser un problema resuelto, se ha incrementado en los últimos años, al punto en que uno de cada tres niños menores de cinco años presentaba desnutrición o sobrepeso. Dicho informe advirtió además que, entre 2000-2016, la proporción de niños y jóvenes ( 5 a 19 años) malnutridos aumentó de uno en diez a casi uno en cinco y que las comunidades más pobres y marginadas eran las que registraban las mayores prevalencias. Es por ello que, en los países en vías de desarrollo como la Argentina, donde la malnutrición se asocia fuertemente con la pobreza, el desarrollo de líneas de investigación que prioricen el análisis integral del problema resulta una necesidad impostergable.

En Argentina, los datos aportados por la $2^{\circ}$ Encuesta Nacional de Nutrición y Salud (Ministerio de Salud y Desarrollo Social, 2019) mostraron que 20,7\% de los niños y adolescentes presentaban sobrepeso; $20,4 \%$, obesidad y 3,7\%, desnutrición. Estos resultados dieron cuenta de que la población infanto-juvenil de nuestro país se encontraría atravesando una etapa dentro del proceso de transición nutricional, en la que el exceso de peso -que contempla tanto al sobrepeso como a la obesidad- es el principal indicador de malnutrición. En coincidencia, trabajos previos realizados en diferentes provincias también han informado que las prevalencias de exceso de peso superaban a las de desnutrición (Garraza, Cesani, Navone y Oyhenart, 2016; Bergel Sanchís, Cesani y Oyhenart, 2017; Lázaro Cuesta et al., 2018; Oyhenart et al., 2018; Bustamante et al., 2019).

El aumento de sobrepeso y obesidad infantil, que se ha registrado a nivel global en los últimos años, ha llevado a considerarlo como uno de los problemas de salud pública más importantes del siglo XXI (World Health Organization, 2017). La obesidad no solo constituye una patología en sí misma, sino que, además, aumenta de manera directa o indirecta el riesgo de padecer enfermedades crónicas tales como diabetes, cáncer, hipertensión arterial, osteoartritis, cardiopatías y accidentes cerebrovasculares (Thompson et al., 2016). Si bien se reconoce que el exceso de peso es de origen multifactorial, los factores determinantes más estudiados se han relacionado con los estilos de vida, y especialmente, con el tipo de alimentación y la actividad física (Wanderley y Ferreira, 2010).

Los hábitos alimentarios dan cuenta de manifestaciones recurrentes de los comportamientos individuales y colectivos respecto del consumo de alimentos que se adoptan, de manera directa o indirectamente, como parte de prácticas socioculturales (Macias, Gordillo y Camacho, 2012). En tal sentido, el entorno familiar resulta de vital importancia, ya que actúa como condicionante de las preferencias y las conductas relacionadas con la alimentación. De igual forma, se ha comprobado la importancia de los padres sobre los niños, niñas y adolescentes en la adquisición de hábitos de actividad física y deportiva. En relación con ello, Abalde-Amoedo y Pino-Juste (2015) destacaron como factor protector, frente a los comportamientos sedentarios de los hijos, la motivación y el acompañamiento de los padres en la realización de actividad física.

En los últimos años, ha crecido el interés por el estudio de los hábitos alimentarios y de la actividad física en el contexto de la urbanización creciente y 
acelerada, por cuanto las características que asumen las ciudades promueven patrones de vida asociados a un mayor sedentarismo y consumo de alimentos no saludables (Moscoso Sánchez, Sánchez García, Martín Rodríguez y Pedrajas Sanz, 2015). Pero, además, en el marco de estos procesos de cambio, la comensalidad -entendida como la acción de comer y beber con otras personas (García Barthe, 2014)- también puede resultar modificada, en particular con relación a aquello que Fischler (2010) definió como "gramática culinaria", es decir, el conjunto de reglas que organizan y definen las formas de obtener, cocinar y consumir los alimentos.

De acuerdo con Ferraro, Zulaica y Echechuri (2013), las ciudades de América Latina se caracterizan por presentar un patrón de "urbanización débil", en el cual la expansión y el crecimiento urbano se dan sin un correlato en el desarrollo de infraestructura y servicios que aseguren una adecuada calidad de vida. Así, a medida que la urbanización avanza, surgen heterogeneidades en las áreas que componen la ciudad y, con ellas, situaciones de desigualdad en los indicadores sociales, nutricionales y de salud de sus habitantes (Torres, 2012). Los nuevos procesos de urbanización tienden a generar movimientos centrífugos desde las ciudades y regiones centrales hacia la periferia (flujos desconcentradores) (Frediani, 2010), lo que da lugar a una interfase de transición en lo urbano y lo rural, conocida como periurbano o periferia urbana. El periurbano es un espacio muy dinámico y heterogéneo, que expone una gran fragilidad ambiental, ya que en él conviven grandes equipamientos e industrias, basurales, asentamientos precarios, cavas, urbanizaciones cerradas, etc. (Frediani, 2009).

El partido de La Plata, ámbito donde se llevó a cabo el presente estudio, es un buen ejemplo del proceso de urbanización antes descrito. Desde la década del ochenta, los límites del casco urbano comenzaron a desbordarse hacia la periferia debido principalmente a dos factores: por un lado, la migración de la clase media y media-alta hacia el sector norte, en busca de espacios con mayor tranquilidad para vivir; y por el otro, la repercusión de la crisis de las décadas del setenta-ochenta-noventa, que produjo el asentamiento en el sector sur de sectores empobrecidos, migrantes intraprovinciales y extranjeros (Frediani, 2009).

Teniendo en cuenta dichas consideraciones, el objetivo del presente estudio fue conocer las prevalencias de exceso de peso y los hábitos vinculados a la alimentación y a la actividad física de niños y niñas residentes en el casco urbano y en la periferia de la ciudad de La Plata (provincia de Buenos Aires, Argentina).

\section{Población y área de estudio}

El estudio se llevó a cabo durante el ciclo lectivo 2019 en tres escuelas públicas (escuelas $\mathrm{N}^{\circ} 43, \mathrm{~N}^{\circ} 40 \mathrm{y} \mathrm{N}^{\circ} 41$ ), seleccionadas por conveniencia y localizadas en el casco urbano (conocido como casco fundacional) y en dos centros comunales (CC) de la periferia de la ciudad de La Plata: Altos de San Lorenzo y San Carlos. La autorización para el ingreso a las escuelas fue gestionada ante la Dirección General de Escuelas de la provincia de Buenos Aires. La población elegible estuvo constituida por todos $\operatorname{los}_{\text {niños }}{ }^{1}$ de 6 a 13 años asistentes a dichos establecimientos educativos. Se excluyeron aquellos escolares que presentaron enfermedad manifiesta al momento del estudio, los que no contaron
1. A fin de facilitar la lectura de este artículo y dado que no hay tratamiento diferencial por sexos en el trabajo, de aquí en más se utilizará el término "niño" para referir a varones y mujeres. 
con el consentimiento informado de los padres o tutores y los que, aun teniéndolo, se rehusaron a participar.

Dentro del casco urbano, la Escuela № 43 se encuentra ubicada en la porción oriental del casco fundacional, en una zona próxima al Paseo del Bosque, donde se localizan, además, distintas facultades de la Universidad Nacional de La Plata. Se trata de una zona residencial de baja densidad situada a corta distancia del centro geográfico y comercial, y con cobertura de todos los servicios públicos esenciales. El nivel socioeconómico (NSE), medido con un índice que integra dimensiones educativas, calidad del material de las viviendas y equipamiento del hogar, se sitúa entre los valores más elevados del partido, y muy próximos a los de las zonas de mayor desarrollo socioeconómico (Alzugaray, Santa María y Peiró, 2019).

Figura 1. Localización de la Escuela $N^{o}$ 43, dentro del casco urbano de la ciudad de La Plata.
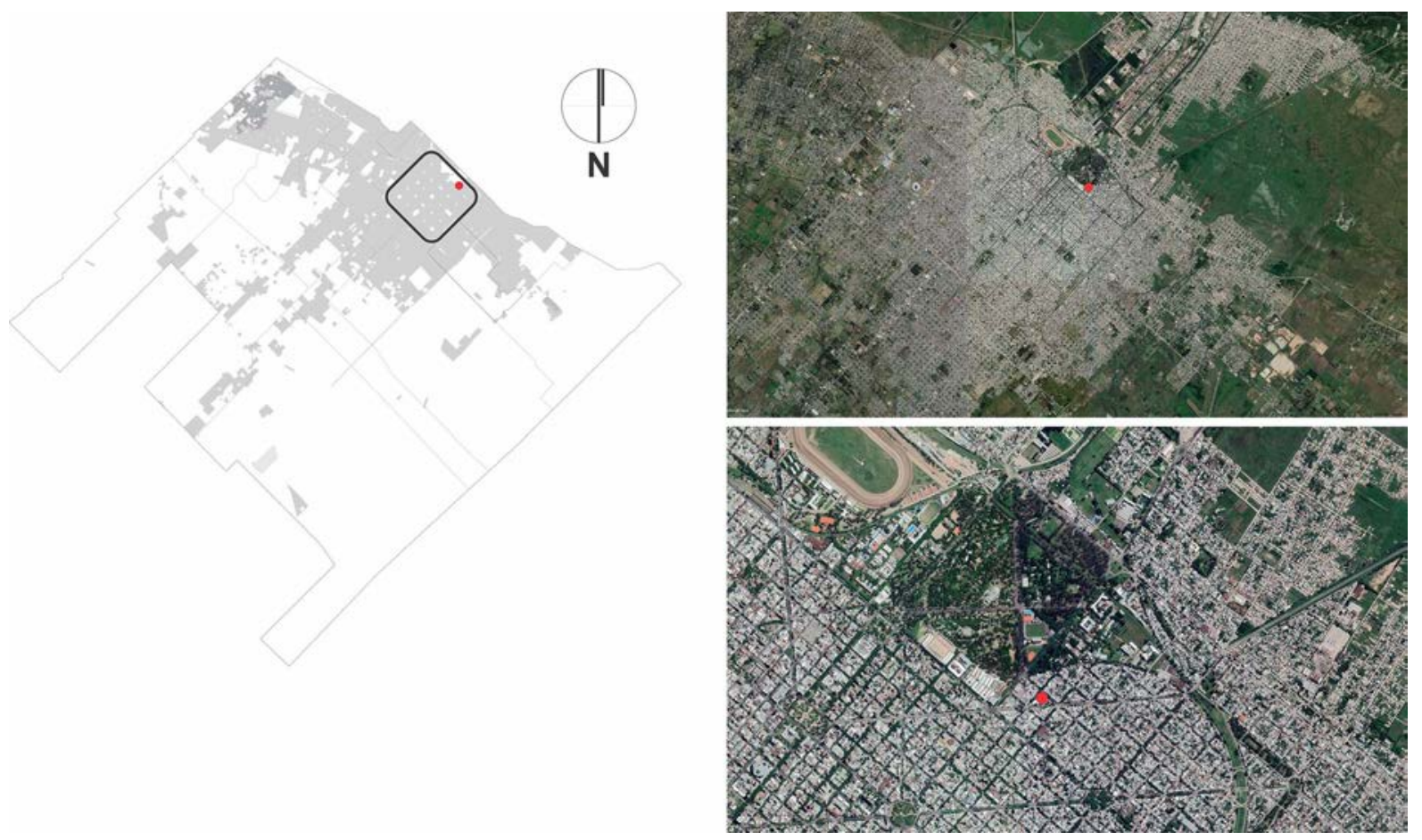

Fuente: Elaboración propia sobre imágenes extraídas de Google Earth.

La Escuela № 40 se encuentra ubicada al este del casco fundacional, en jurisdicción del CC Altos de San Lorenzo. Este centro comunal, lindero con el casco fundacional a través de la Avenida 72, se extiende en el extremo opuesto al sector de mayor desarrollo del partido, lo que se traduce en ciertas condiciones de marginalidad. Se caracteriza por ser una zona residencial de baja densidad en la que, según Rodríguez Tarducci (2018), se han instalado, durante las últimas décadas, ocho asentamientos informales donde residen entre 100 y 1000 familias, de las cuales, el $8 \%$ tienen acceso al 100\% de los servicios básicos, el $38 \%$ no tiene acceso a ningún servicio, mientras que el $12 \%$, el $38 \%$ y el $12 \%$ respectivamente, tienen acceso solo a 1, 2, y 3 servicios esenciales básicos. De acuerdo con Alzugaray et al. (2019), el NSE en una pequeña porción de su territorio, ubicada en la zona más próxima a su límite con el casco fundacional, 
se sitúa en valores medios; mientras que en la mayor parte de su superficie predominan valores muy bajos (figura 2).

Figura 2. Localización de la Escuela № 40, dentro del centro comunal Altos de San Lorenzo.
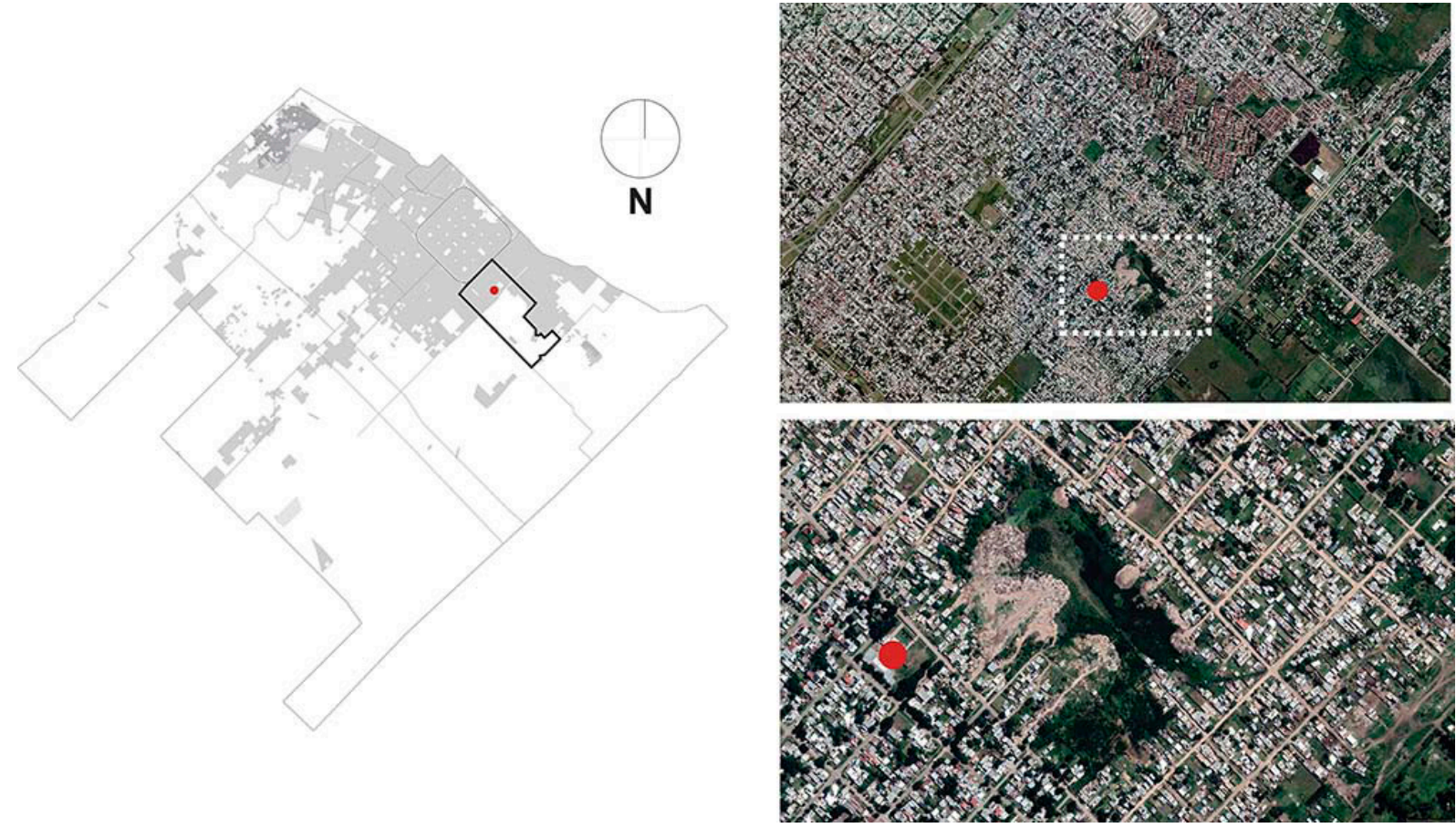

Fuente: Elaboración propia sobre imágenes extraídas de Google Earth.

Por último, la Escuela № 41, ubicada al sudoeste del casco fundacional, en jurisdicción del centro comunal San Carlos, lindero con el casco urbano, se sitúa sobre la Avenida 520, principal vía de comunicación del centro de la ciudad con la zona sudoeste del partido. Se caracteriza por la coexistencia de usos del suelo residencial y productivo. Se extiende en una zona en la que, en el pasado, predominó la producción hortícola intensiva, actividad progresivamente desplazada por el crecimiento demográfico de la ciudad. De acuerdo con Rodríguez Tarducci (2018), en las últimas décadas se han instalado nueve asentamientos informales, donde residen entre 100 y 1000 familias, de las cuales, el $9 \%$ tienen acceso al 100\% de los servicios básicos, el $67 \%$ no tiene acceso a ningún servicio, mientras que el 22\%, y $11 \%$ tienen acceso solo a 2 y 3 servicios esenciales básicos. El NSE se sitúa en valores medios en una pequeña porción de su territorio, en valores bajos en la mayoría de su extensión, y muy bajos al sur, donde limita con el CC de Melchor Romero, que presenta los NSE más bajos del partido de La Plata (Alzugaray et al., 2019) (figura 3). 
Figura 3. Localización de la Escuela № 41, dentro del centro comunal San Carlos.

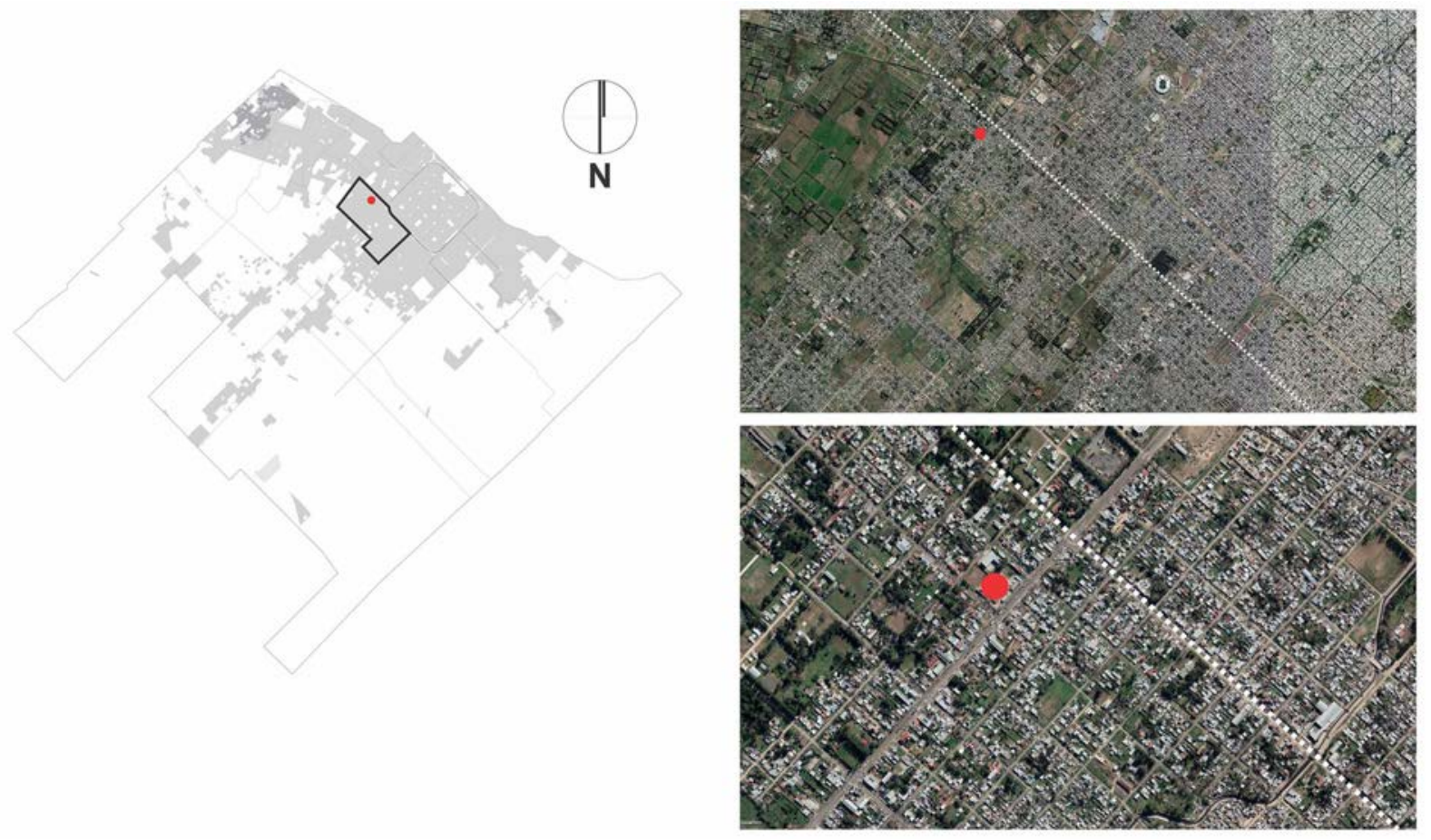

Fuente: Elaboración propia sobre imágenes extraídas de Google Earth.

\section{Metodología}

El estudio fue de tipo transversal e incluyó tres ejes de análisis: a) análisis socioambiental; b) análisis antropométrico y determinación del estado nutricional; y c) análisis de hábitos alimentarios y actividad física.

\section{Análisis socioambiental}

Se realizó una encuesta estructurada y autoadministrada que fue respondida por los padres o tutores de los escolares participantes en el estudio. Se relevaron factores a escala de la vivienda: régimen de tenencia y materiales constructivos; del combustible usado para cocinar y aspectos vinculados con la familia: presencia de hacinamiento crítico (más de tres individuos por cuarto), nivel educativo y tipo de empleo de los progenitores y situación socioeconómica familiar, valorada por las ayudas monetaria y/o alimentaria a cargo del Estado u otros organismos no gubernamentales y por las prácticas de autoproducción de alimentos, huerta y cría de animales (Oyhenart et al., 2008).

\section{Análisis antropométrico y determinación del estado nutricional}

Se realizó un estudio antropométrico transversal en escolares de ambos sexos, con edades comprendidas entre los 6 y los 13 años. Siguiendo protocolos estandarizados, se relevaron: 1) peso corporal (en kilogramos), empleando una balanza digital portátil (100 g de precisión), que se calibró al inicio de cada sesión; en todos los casos los niños vistieron ropa liviana cuyo peso se descontó del peso total; y 2) talla (en centímetros) utilizando un antropómetro vertical 
(1 mm de precisión) con el niño descalzo, erguido y con la cabeza orientada en el plano de Frankfort (Lohman, Roche y Martorell, 1988).

Con los valores de peso y talla se calculó el índice de masa corporal [IMC= peso $(\mathrm{kg}) /$ talla $(\mathrm{m} 2)]$. El estado nutricional se determinó empleando la referencia y puntos de corte propuestos por la Organización Mundial de la Salud (de Onis et al., 2007) mediante el programa informático WHO Antroplus v. 1.0.3. Los escolares con bajo peso/edad, baja talla/edad y bajo IMC/edad (puntajes z P/E, zT/E, zIMC/edad <-2), fueron considerados desnutridos (D); y aquellos con sobrepeso (puntajes z IMC/E $>1 y \leq 2$ ) y obesidad (puntaje z IMC/E $>2$ ), con exceso de peso (EP). Los niños que no quedaron comprendidos en las categorías antes mencionadas se consideraron con estado nutricional adecuado (ENA).

\section{Análisis de hábitos alimentarios y actividad física}

Para el análisis de los hábitos alimentarios y de actividad física se tomó como referencia el cuestionario de Nutrición Familiar y Actividad Física (FNPA) (Ihmels, Welk, Eisenmann y Nusser, 2009), adaptado para la mejor comprensión de las preguntas. El cuestionario fue autoadministrado y respondido por los padres o tutores de los escolares y se utilizó para obtener información detallada sobre los hábitos alimentarios y de actividad física promovidos desde el hogar hacia los niños.

El instrumento aplicado indagó sobre distintos factores de riesgo vinculados con los hábitos de la familia y de los hijos, tales como uso del televisor, celular u otros dispositivos electrónicos durante las comidas y en la habitación; tipo de alimentación y hábito de desayuno. Además, se preguntó sobre la cantidad de horas de sueño del niño, el control de estas por parte de los padres, la realización de actividad física por parte del niño y el incentivo y el acompañamiento por parte de los padres para su realización.

El cuestionario incluyó 20 ítems con una escala de respuesta tipo Likert: nunca; a veces (de una a tres veces por semana); casi siempre (de cuatro a seis veces por semana); siempre (todos los días de la semana). Las respuestas de cada ítem fueron codificadas como 1, 2, 3 o 4; el valor más bajo les fue otorgado a las respuestas relacionadas con hábitos "menos saludables".

Además del análisis particular de cada ítem, se obtuvo la puntuación total del cuestionario a partir de la suma de las puntuaciones parciales, cuyo valor pudo oscilar entre 1 y 80 . La puntuación total alta equivale a hábitos saludables de la familia y del niño con relación a la alimentación y a la actividad física en contraposición a la puntuación total baja, que refleja un entorno familiar obesogénico y comportamientos que aumentan el riesgo de sobrepeso y obesidad (Ihmels et al., 2009).

\section{Análisis de los datos}

De la matrícula correspondiente a niños de 6 a 13 años consignada en los registros de las escuelas incluidas en el estudio (n: 1541), se relevaron el peso y la talla de 558 escolares y se obtuvieron 514 cuestionarios, de los cuales solo el $32 \%$ fueron respondidos en su totalidad. Una vez excluidos los protocolos incompletos, la muestra quedó conformada por 163 escolares (varones: 75 y mujeres: 88), distribuidos por escuelas de la siguiente manera: casco urbano: 53; Altos de San Lorenzo: 47; San Carlos: 63. Sobre esta muestra, se calcularon 
prevalencias generales de estado nutricional (ENA, EP y D) discretizadas por centro comunal de residencia.

Luego, y atendiendo el objetivo del estudio, los escolares que presentaron desnutrición (n: 9) -bajo peso/edad, baja talla/edad y bajo IMC/edad-fueron excluidos, y el análisis de las características socioambientales de residencia y de los hábitos alimentarios y de actividad física se realizó solo para los niños que presentaron ENA y EP: 154 escolares (varones: 71 varones y mujeres: 83), distribuidos por escuelas de la siguiente manera: casco urbano: 53; Altos de San Lorenzo: 43; San Carlos: 58.

El análisis de los hábitos de alimentación y de actividad física se efectuó en dos niveles: a) sobre cada ítem del cuestionario; y b) sobre la puntuación total obtenida para todo el cuestionario. Para ello, se estimaron parámetros descriptivos de tendencia central y de dispersión; se realizaron pruebas de bondad de ajuste de Kolmogorov-Smirnov y pruebas U de Mann-Whitney y Kruskal-Wallis para detectar diferencias de acuerdo con el estado nutricional y con el centro comunal de residencia, respectivamente.

Todo el análisis estadístico se realizó empleando el software SPSS 15.0.

\section{Consideraciones éticas}

El protocolo de este trabajo fue aprobado por el Comité de Bioética de la Universidad Nacional de La Plata. La investigación se desarrolló conforme con los principios proclamados en la Declaración Universal de los Derechos Humanos de 1948; las normas éticas instituidas por el Código de Nüremberg en 1947; la Declaración de Helsinski de 1964 y sus sucesivas enmiendas y clarificaciones; y la Ley Nacional 25.326, modificada por Ley 26.343 de protección de datos personales y su Reglamentación № 1.558/01.

\section{Resultados}

\section{Condiciones socioambientales de residencia}

El análisis socioambiental de residencia de las familias y los escolares dio cuenta de que aproximadamente el $80 \%$ de las familias eran propietarias y el $20 \%$ residían en viviendas prestadas o alquiladas. En San Carlos, había mayor porcentaje de viviendas prestadas. El material empleado en la construcción de las paredes era, principalmente, la mampostería de ladrillo; no obstante, en Altos de San Lorenzo y en San Carlos, el 24\% y el 29\% de las viviendas tenían, respectivamente, paredes de madera; y en este último centro comunal, el 5\% era de chapa. El hacinamiento crítico fue de $27 \%$ en toda la población y el mayor valor desagregado (37\%) correspondió a Altos de San Lorenzo (Tabla 1).

El combustible mayormente utilizado para cocinar fue el gas envasado (76\%); con valores menores en el casco urbano y mayores en Altos de San Lorenzo y San Carlos; en esta última zona se adicionaba el empleo de leña (Tabla 1).

Aproximadamente el $29 \%$ de la población recibía ayuda monetaria; el $2 \%$, ayuda alimentaria; el $7 \%$ disponía de huerta; y el $2 \%$ criaba animales. Diferenciadas por centros comunales, las familias residentes en San Carlos 
fueron las que tuvieron más asistencia por parte del Estado, y las de Altos de San Lorenzo, las que tenían huertas y animales para autoconsumo (Tabla 1).

En general, el mayor nivel de instrucción de los padres y de las madres fue el primario, incompleto y completo, aunque en el caso de la madre los valores porcentuales fueron similares a los del secundario completo. El nivel terciario/ universitario completo fue mayor en las madres. Por último, en la población en general, y con porcentajes similares, los padres eran principalmente empleados formalizados o realizaban changas. Esto fue muy notorio en las familias residentes en Altos de San Lorenzo, seguidas por las de San Carlos, las cuales presentaron, además, el mayor porcentaje de desocupación. En el caso de las madres, la mitad eran amas de casa; San Carlos fue el centro comunal que registró la mayor frecuencia, tanto de amas de casa como de desocupadas (Tabla 1).

Tabla 1. Condiciones socioambientales de residencia de la población total y por centros comunales

\begin{tabular}{|c|c|c|c|c|}
\hline Variable & $\begin{array}{l}\text { TOTAL (n: } \\
\text { 154) }\end{array}$ & $\begin{array}{c}\text { Casco } \\
\text { urbano }(\mathrm{n}: \\
53)\end{array}$ & $\begin{array}{c}\text { Altos de San } \\
\text { Lorenzo } \\
\text { (n: 43) }\end{array}$ & $\begin{array}{c}\text { San Carlos } \\
\text { (n: } 58\end{array}$ \\
\hline \multicolumn{5}{|l|}{ Tenencia de vivienda } \\
\hline Propia & 76,7 & 79,2 & 89,4 & 65,2 \\
\hline Alquilada & 9,8 & 15,1 & 6,4 & 7,9 \\
\hline Prestada & 12,9 & 5,7 & 4,4 & 25,9 \\
\hline NS/NC & 0,6 & - & - & 0,8 \\
\hline \multicolumn{5}{|c|}{ Construcción de la vivienda* } \\
\hline Ladrillo & 84,7 & 94,3 & 80,9 & 79,4 \\
\hline Chapa & 3,1 & 3,8 & - & 4,8 \\
\hline Madera & 19,6 & 5,7 & 23,4 & 28,6 \\
\hline Hacinamiento crítico & 26,7 & 25,0 & 37,0 & 20,6 \\
\hline \multicolumn{5}{|c|}{ Combustible para cocinar* } \\
\hline Gas natural & 19,6 & 43,4 & 2,1 & 12,7 \\
\hline Garrafa & 76,1 & 58,5 & 89,4 & 81,0 \\
\hline Leña & 1,8 & - & 6,4 & - \\
\hline Ayuda monetaria & 28,8 & 26,4 & 27,7 & 31,7 \\
\hline Ayuda alimentaria & 1,8 & 1,9 & - & 3,2 \\
\hline Huerta & 7,4 & 9,4 & 12,8 & 1,6 \\
\hline Cría de animales & 6,1 & 1,9 & 17,0 & 1,6 \\
\hline \multicolumn{5}{|c|}{ Nivel de instrucción paterno } \\
\hline Primario incompleto & 19,6 & 3,8 & 34,1 & 22,2 \\
\hline Primario completo & 27,6 & 22,6 & 25,5 & 33,3 \\
\hline Secundario completo & 34,4 & 52,8 & 25,5 & 25,4 \\
\hline $\begin{array}{l}\text { Terciario/Universitario } \\
\text { completo }\end{array}$ & 6,1 & 15,1 & 4,3 & - \\
\hline
\end{tabular}




\begin{tabular}{|c|c|c|c|c|}
\hline Variable & $\begin{array}{l}\text { TOTAL (n: } \\
\text { 154) }\end{array}$ & $\begin{array}{c}\text { Casco } \\
\text { urbano }(\mathrm{n}: \\
53) \\
\end{array}$ & $\begin{array}{l}\text { Altos de San } \\
\text { Lorenzo } \\
(n: 43)\end{array}$ & $\begin{array}{c}\text { San Carlos } \\
\text { (n: } 58\end{array}$ \\
\hline $\mathrm{NS} / \mathrm{NC}$ & 12,3 & 5,7 & 10,6 & 19,1 \\
\hline \multicolumn{5}{|c|}{ Nivel de instrucción materno } \\
\hline Primario incompleto & 15,3 & 9,4 & 25,5 & 12,7 \\
\hline Primario completo & 23,9 & 17,0 & 23,4 & 30,2 \\
\hline Secundario completo & 39,9 & 34,0 & 38,3 & 46,0 \\
\hline $\begin{array}{l}\text { Terciario/Universitario } \\
\text { completo }\end{array}$ & 14,2 & 34,0 & 4,3 & 4,8 \\
\hline NS/NC & 6,7 & 5,6 & 8,5 & 6,3 \\
\hline \multicolumn{5}{|l|}{ Trabajo paterno* } \\
\hline Empleado & 3,5 & 45,3 & 19,1 & 38,1 \\
\hline Autónomo & 17,2 & 17,0 & 19,1 & 15,9 \\
\hline Hace changas & 34,4 & 32,1 & 48,9 & 25,4 \\
\hline Desocupado & 8,6 & 5,7 & 8,5 & 11,1 \\
\hline Jubilado & 0,6 & - & 2,1 & - \\
\hline \multicolumn{5}{|l|}{ Trabajo materno* } \\
\hline Ama de casa & 50,9 & 39,6 & 53,2 & 58,7 \\
\hline Empleada & 21,5 & 32,1 & 19,1 & 14,3 \\
\hline Autónoma & 11,0 & 13,2 & 6,4 & 12,7 \\
\hline Desocupada & 7,4 & 9,4 & - & 11,1 \\
\hline Hace changas & 9,8 & 13,2 & 8,5 & 7,9 \\
\hline Jubilada & 3,1 & - & 8,5 & 1,6 \\
\hline
\end{tabular}

Fuente: Elaboración propia en base a datos obtenidos en el presente estudio. NS/NC: no sabe, no contesta *Incluyen respuestas múltiples (total $>100 \%$ )

\section{Estado nutricional antropométrico}

El análisis antropométrico indicó que el 50,3\% de los escolares presentó ENA; $5,5 \%$, desnutrición ( $1,2 \%$ bajo peso/edad, $3,7 \%$ baja talla/edad, $0,6 \%$ bajo IMC/ edad); en tanto que el $44,2 \%$, EP, siendo el sobrepeso (27,6\%) más frecuente que la obesidad $(16,6 \%)$.

En la Tabla 2 se presentan las prevalencias de estado nutricional de acuerdo con el lugar de residencia. Altos de San Lorenzo presentó la prevalencia más alta de escolares con desnutrición y la más baja de exceso de peso. Por el contrario, San Carlos evidenció la mayor prevalencia de EP y la menor de ENA. Por último, el casco urbano mostró una situación intermedia para el EP y la mayor prevalencia de ENA, en tanto que no registró casos de desnutrición (Tabla 2). 
Tabla 2. Prevalencias de estado nutricional de acuerdo con el lugar de residencia

\begin{tabular}{|c|c|c|c|}
\hline Lugar de residencia & Estado nutricional & $\begin{array}{c}\text { Prevalencia } \\
\text { (\%) }\end{array}$ & $\begin{array}{c}\text { Número de } \\
\text { casos } \\
\mathrm{n}: 163\end{array}$ \\
\hline \multirow{3}{*}{ Casco urbano } & Adecuado & 56,6 & 30 \\
\hline & Desnutrición & - & \\
\hline & Exceso de peso & 43,4 & 23 \\
\hline \multirow{3}{*}{ Altos de San Lorenzo } & Adecuado & 53,2 & 25 \\
\hline & Desnutrición & 8,5 & 4 \\
\hline & Exceso de peso & 38,3 & 18 \\
\hline \multirow{3}{*}{ San Carlos } & Adecuado & 42,9 & 27 \\
\hline & Desnutrición & 7,9 & 5 \\
\hline & Exceso de peso & 49,2 & 31 \\
\hline
\end{tabular}

Fuente: Elaboración propia en base a datos obtenidos en el presente estudio.

\section{Hábitos alimentarios y de actividad física}

En la figura 4 se presentan los valores medios obtenidos para cada uno de los 20 ítems del cuestionario de hábitos alimentarios y de actividad física en la muestra total. Se observa que, en general, las preguntas relacionadas con los hábitos familiares y de control por parte de los padres obtuvieron puntuaciones más altas que las correspondientes a los hábitos del niño. El uso poco frecuente del celular durante las comidas, el control por parte de los padres del horario de descanso de los niños y de la duración mínima de 8 horas de sueño fueron los ítems mejor puntuados. Por el contrario, la realización de actividad física junto a los hijos, el consumo de alimentos con alto contenido de hidratos de carbono y el hacer deportes extraescolares fueron las preguntas con puntuaciones menores. 
Figura 4. Valores medios obtenidos para la muestra total de los 20 items del cuestionario de hábitos alimentarios y de actividad física.

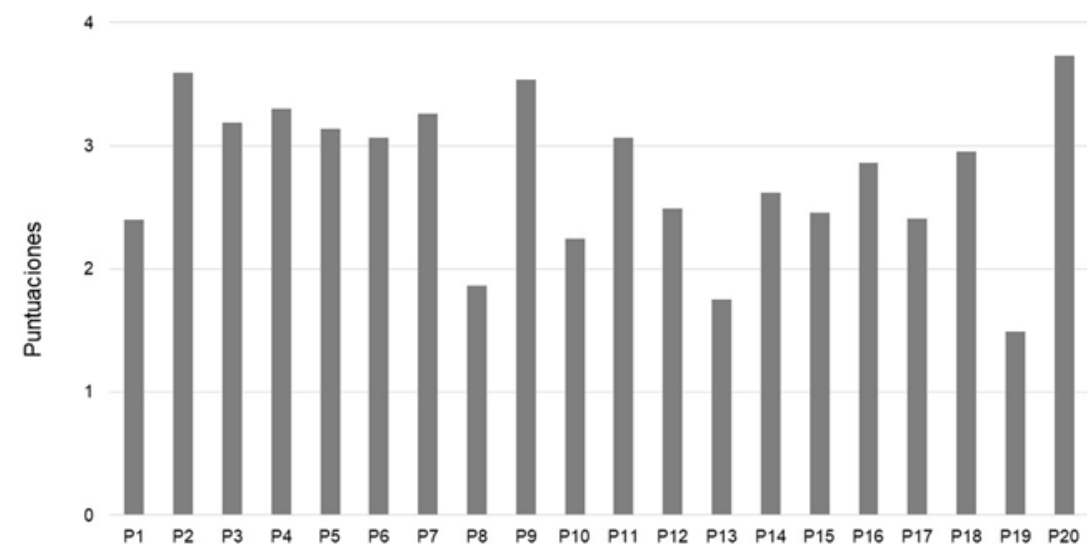

Referencia: Preguntas referidas al entorno familiar: P1: ¿Miran televisión cuando comen?; P2: ¿¿Usan el teléfono celular mientras comen?; P3. ¿Comen "comida rápida"?; P4. ¿Consumen alimentos precocidos?; P5. ¿Controlan la cantidad de golosinas, papas fritas y galletitas que come su hijo?; P6. ¿Controlan el tiempo que su hijo mira televisión o usa la computadora, videojuegos o celular?; P7. ¿incentivan a su hijo a realizar actividad física?; P8. ¿Realizan alguna actividad física junto a su hijo?; P9. ¿Controlan el horario en que su hijo se va a dormir?; Preguntas referidas a hábitos del niño: P10. ¿Desayuna en casa?; P11. ¿Come frutas y verduras?; P12. ¿Come huevos?; P13. ¿Come pan, arroz, fideos, polenta?; P14. ¿Come caramelos, alfajores, chocolates, etc.?; P15. ¿Toma gaseosas o bebidas azucaradas?; P16. ¿Come entre las comidas principales sándwiches, golosinas, galletitas?; 17. ¿Mira televisión, usa la computadora, celular o videojuegos en el dormitorio?; P18. ¿Hace actividad física cuando tiene tiempo libre?; P19. ¿Hace deportes o actividades organizadas por un profesor fuera de la escuela?; P20. ¿Duerme por la noche un mínimo de

8 horas?

Fuente: Elaboración propia en base a datos obtenidos en el presente estudio.

En la Tabla 3 se muestran los promedios, desvíos estándar, valores mínimos y máximos y valores U de Mann-Whitney y Kruskal-Wallis del puntaje total del cuestionario discretizado por estado nutricional y lugar de residencia. En todos los casos, las puntuaciones medias fueron mayores a 50, con diferencias no significativas entre estados nutricionales y significativas entre centros comunales (Tabla 3). En relación con esto último, las pruebas post hoc indicaron que los escolares de Altos de San Lorenzo obtuvieron puntuaciones más altas que sus pares residentes en el casco urbano y en San Carlos (Tabla 4). 
Tabla 3. Media, desvío estándar y valores mínimos y máximos de la puntuación total obtenida en el cuestionario de hábitos alimentarios y de actividad física. Comparaciones por estado nutricional y lugar de residencia

\begin{tabular}{l|c|c|c|c|c}
\hline & Media & $\begin{array}{c}\text { Desvío } \\
\text { estándar }\end{array}$ & $\begin{array}{c}\text { Valor } \\
\text { mínimo }\end{array}$ & $\begin{array}{c}\text { Valor } \\
\text { máximo }\end{array}$ & $p$ \\
\hline Estado nutricional & & & & & $0^{\circ}, 80^{\mathrm{a}}$ \\
\hline Adecuado & 55 & 7 & 36 & 68 & \\
\hline Exceso de peso & 56 & 6 & 46 & 70 & \\
\hline Lugar de residencia & 54 & 6 & 38 & 68 & \\
\hline Casco urbano & 58 & 6 & 44 & 70 & \\
\hline Altos de San Lorenzo & 54 & 7 & 36 & 65 & \\
\hline San Carlos & & & & & \\
\hline
\end{tabular}

a Resultado U de Mann-Whitney. b Resultado Kruskal-Wallis. Fuente: Elaboración propia en base a datos obtenidos en el presente estudio.

Tabla 4. Pruebas post hoc para comparar la puntuación total del cuestionario de hábitos alimentarios y actividad física de acuerdo con el lugar de residencia

\begin{tabular}{|c|c|c|c|c|}
\hline \multirow{2}{*}{ Lugar de residencia } & \multirow{2}{*}{$\begin{array}{c}\text { Diferencia de } \\
\text { medias }\end{array}$} & \multirow{2}{*}{$\mathbf{p}$} & \multicolumn{2}{|c|}{$\begin{array}{c}\text { Intervalo de confianza } \\
\text { al } 95 \%\end{array}$} \\
\hline & & & $\begin{array}{l}\text { Límite } \\
\text { inferior }\end{array}$ & $\begin{array}{l}\text { Límite } \\
\text { superior }\end{array}$ \\
\hline $\begin{array}{l}\text { Casco urbano vs. Altos de } \\
\text { San Lorenzo }\end{array}$ & $-3,92$ & 0,002 & $-6,97$ & $-0,87$ \\
\hline $\begin{array}{l}\text { Casco urbano vs. San } \\
\text { Carlos }\end{array}$ & $-0,173$ & 0,556 & $-3,01$ & 2,66 \\
\hline $\begin{array}{l}\text { Altos de San Lorenzo vs. } \\
\text { San Carlos }\end{array}$ & 3,747 & 0,007 & 0,81 & 6,68 \\
\hline
\end{tabular}

Fuente: Elaboración propia en base a datos obtenidos en el presente estudio.

Por último, la comparación de los puntajes medios obtenidos para cada ítem del cuestionario mediante el test de Kruskall Wallis indicó diferencias significativas entre CC en 6 de las 20 preguntas. Se evidenciaron diferencias de acuerdo con el lugar de residencia; fueron aquellas vinculadas al hábito de mirar televisión y usar teléfono celular durante las comidas, desayunar en la casa, consumir frutas, verduras y alimentos ricos en hidratos de carbono y comer entre las comidas principales sándwiches, golosinas, galletitas y otros alimentos (figura 5). Por el contrario, los puntajes obtenidos en las preguntas relativas a los hábitos vinculados a la actividad física o al sueño fueron similares en los tres CC ( $p>0.05)$. 
Figura 5. Comparaciones de acuerdo con el lugar de residencia (Test de Kruskal-Wallis). Puntuaciones medias de los ítems de cuestionario hábitos alimentarios y actividad física que mostraron diferencias significativas.

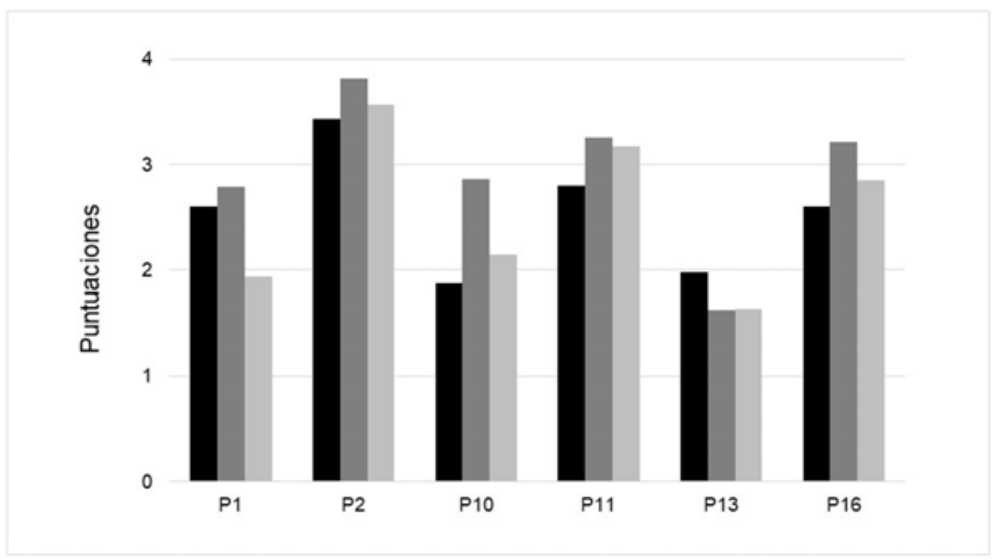

Referencias: Barras negras: Casco Urbano, Barras gris oscuro: Altos de San Lorenzo y Barras gris claro: San Carlos. Preguntas referidas al entorno familiar: P1: ¿Miran televisión cuando comen?; P2: ¿Usan el teléfono celular mientras comen?; Preguntas referidas a hábitos del niño: P10: ¿Desayuna en casa?; P11: ¿Come frutas y verduras?; P13: ¿Come pan, arroz, fideos, polenta?; P16: ¿Come entre las comidas principales sándwiches, golosinas, galletitas? Fuente: Elaboración propia en base a datos obtenidos en el presente estudio.

\section{Discusión}

El incremento del sobrepeso y la obesidad infantil registrado en los últimos años ha impulsado la realización de innumerables trabajos que abordan el problema desde diferentes perspectivas. Desde la antropología, el presente estudio aporta evidencia acerca de las elevadas prevalencias de exceso de peso y de los hábitos vinculados a la alimentación y a la actividad física de los niños residentes en zonas urbanas y periurbanas de la ciudad de La Plata.

Aproximadamente cuatro de cada diez escolares presentaron exceso de peso, siendo el sobrepeso más frecuente que la obesidad. Los valores encontrados fueron similares a los recientemente informados para otras poblaciones del país (Ministerio de Salud y Desarrollo Social, 2019; Cordero y Cesani, 2019; Navazo, Oyhenart y Dahinten, 2019) y dan cuenta de la magnitud del problema. Los resultados reflejan, además, el estadio avanzado de transición nutricional en el que se encuentra la población analizada, que evidencia altas prevalencias de sobrepeso y obesidad, en coexistencia con desnutrición.

Por otra parte, se observaron diferencias de acuerdo con el lugar de residencia. Por ejemplo, San Carlos presentó casi un $6 \%$ más de casos de exceso de peso que el casco urbano, en tanto que Altos de San Lorenzo fue el centro comunal con menor prevalencia. Si bien el exceso de peso es, en última instancia, la manifestación del balance positivo entre la ingesta y el gasto de energía, su origen multicausal involucra la interacción de factores biológicos y sociales. En tal sentido, cobra importancia el concepto de entorno obesogénico, definido como la suma de influencias ambientales que propicia hábitos y estilos de vida poco saludables y conducen al aumento de obesidad (Swinburn, Egger y Raza, 1999). 
En el caso de los niños, son los padres o cuidadores los que en mayor medida establecen el entorno físico y social en el que aquellos crecen y se desarrollan. De acuerdo con Boswell, Byrne y Davies (2019), el entorno familiar es de gran importancia, ya que es el primer nicho ecológico del niño y actúa como promotor o inhibidor de hábitos y conductas obesogénicas que pueden perdurar durante toda la vida. Los padres trasmiten a los hijos valores y prácticas socioculturales en torno a la alimentación y a la actividad física, y es por esta razón que gran parte de las intervenciones preventivas se focalizan en el microambiente familiar (Ministerio de Salud de la Nación, 2013). Se ha observado, por ejemplo, que la probabilidad de que los hijos sean activos aumenta dos veces cuando la madre lo es; en tanto que si ambos padres lo son, la posibilidad es 5,8 veces mayor (Moore et al., 1991). Asimismo, se ha demostrado que los estilos de crianza indulgentes se relacionan con valores de IMC elevados y con conductas de alimentación poco saludables de los hijos (Johnson, Welk, Saint-Maurice e Imhels, 2012).

Con relación a ello, y a pesar de las altas prevalencias de sobrepeso y obesidad encontradas, los resultados indicaron que, en general, la población presentaba hábitos relativamente saludables. El control de los padres estuvo especialmente presente en lo referido al uso del celular durante las comidas, las horas de permanencia frente al televisor, la cantidad de golosinas, snacks y galletitas consumidas y el horario de descanso de los niños. Asimismo, en el ámbito familiar, el consumo de comidas rápidas y alimentos precocidos era poco habitual, y los niños comían, frecuentemente, frutas y verduras. No obstante, también se registró que, en general, la alimentación diaria incluía comidas o alimentos ricos en hidratos de carbono, como pan, arroz, fideos y polenta. Por otra parte, y en relación con la actividad física, se observó que, si bien los padres incentivaban a los niños a realizarla, no compartían esta práctica con ellos. En concordancia con estos resultados, Pocock, Trivedi, Wills, Bunn y Magnusson (2009), a partir de una revisión sistemática de estudios cualitativos, informaron que, en general, los padres consideraban importante actuar como modelos positivos de las conductas de los hijos, aunque también argumentaban que, por falta de tiempo, muchas veces esto no sucedía. Dichos autores encontraron, además, que gran parte de padres creían que el hecho de motivar a los hijos a ser activos era condición suficiente para que ellos incorporaran dicho hábito.

Por otra parte, solo la mitad de los escolares participantes de nuestro estudio solían jugar al aire libre, andar en bicicleta, caminar o correr en su tiempo de ocio, y muy pocos hacían deportes o actividades organizadas por un profesor fuera del ámbito escolar. Si consideramos las características socioeconómicas familiares, esta situación podría vincularse a la falta de recursos para solventar la realización de deportes en ámbitos privados, o bien a la inexistencia de entidades públicas que ofrecieran dichos servicios de forma gratuita. En tal sentido, cabe mencionar que solo el $50 \%$ de los padres contaban con empleos formalizados, en tanto que más del $40 \%$ realizaban changas o estaban desocupados. A su vez, la mitad de las madres eran amas de casa, solo el 30\% tenía empleo o era trabajadora autónoma, y el 10\% hacían changas. La precarización laboral fue aún más notoria en las familias residentes en la periferia de la ciudad, donde, además, se presentó el mayor porcentaje de desocupación paterna; situación que fue particularmente notoria en San Carlos.

De acuerdo con Allén (2003), el espacio periurbano suele presentarse como una interfase ecológica, caracterizada por la disminución de los servicios propios del sistema urbano y el debilitamiento de los servicios cumplidos por los sistemas rurales y naturales. Desde el punto de vista socioeconómico, 
también presenta características de mosaico, ya que el proceso de urbanización continuo, aunque disperso, responde en muchos casos a la especulación inmobiliaria, a los cambios de uso de suelo hacia actividades de mayor productividad y/o a la rentabilidad y la emergencia de actividades informales. En consecuencia, la composición social de los sistemas periurbanos es extremadamente heterogénea y dinámica. En concordancia con ello, el espacio periurbano analizado, lejos de comportarse como un todo homogéneo, evidenció marcadas diferencias socioambientales, reflejadas, principalmente, en la propiedad y en las características constructivas de la vivienda, el hacinamiento crítico, el acceso a servicios públicos, la práctica de huerta y cría de animales para el autoconsumo y el bajo nivel educativo y empleo de los padres y de las madres. Dicha heterogeneidad podría explicar, en parte, las diferencias de sobrepeso y obesidad encontradas en ambos CC periurbanos.

En tal sentido, consideramos importante destacar que, si bien los hábitos alimentarios y de actividad física resultaron ser similares entre los niños con estado nutricional adecuado y con exceso de peso, fueron los escolares de Altos de San Lorenzo quienes, además de presentar las menores prevalencias de sobrepeso y obesidad, evidenciaron hábitos y conductas más saludables. En el entorno familiar, se observó menor uso del televisor y del celular durante las comidas y, en relación con la alimentación de los niños, se registró el hábito diario de desayunar en el hogar y el consumo frecuente de frutas y verduras. Al respecto, es posible que el desarrollo de huertas familiares -práctica habitual en esta localidad-se relacione con el mayor consumo de vegetales frescos por parte de los niños.

Por el contrario, en el casco urbano -y especialmente en el centro comunal periurbano San Carlos-, el uso del televisor durante las comidas, la omisión del desayuno y el consumo de alimentos ricos en hidratos de carbono fueron algunas de las conductas obesogénicas más frecuentemente observadas. Autores como Spurrier, Magarey, Golley, Curnow y Sawyer (2008) y Koh et al. (2014) han informado que el uso del televisor durante las comidas y la baja disponibilidad de frutas y verduras en el hogar se asocian a conductas obesogénicas. Por otra parte, la omisión del desayuno también es considerado un factor de riesgo de obesidad, por cuanto induce al consumo de alimentos entre comidas -generalmente de bajo valor nutricional y de alta densidad calórica-y de almuerzos más abundantes (Wijtzes et al., 2016). Coincidentemente, junto con la omisión del desayuno, los resultados obtenidos dieron cuenta de que el picoteo o "snacking" -que refiere a la ingesta de galletitas, golosinas u otros alimentos por fuera de las comidas estructuradas- era frecuente en los escolares del casco urbano y de San Carlos. Esta práctica es señalada como uno de los grandes problemas de la alimentación actual y constituye, según Fischler (2010), el paso de la "comensalidad" a la "individualidad" y el surgimiento de la "gastro-anomia", es decir, la libertad de comer fuera de los requisitos y de las reglas de sociabilidad alimentaria.

El presente estudio aporta información novedosa acerca de un problema de salud pública actual para los niños de La Plata. En este sentido, se revela que los procesos de crecimiento y desarrollo están fuertemente condicionados por el estado nutricional, el que, a su vez, se relaciona con los hábitos alimentarios y la actividad física, atendiendo no solo a las conductas individuales, sino también a las del entorno familiar. Asimismo, otra aportación que arroja este trabajo es que tanto el sobrepeso como la obesidad expresan su naturaleza compleja, es decir, responden a factores diversos. Los resultados obtenidos podrían ser utilizados como potentes insumos en el diseño de las políticas 
públicas, a dictarse por los organismos competentes, con el objetivo de referirlas a contextos específicos y destinarlas a mejorar la calidad de vida de los niños y sus entornos familiares.

\section{Conclusiones}

En el casco urbano y en la periferia de la ciudad de La Plata, se registran elevadas prevalencias de sobrepeso y obesidad infantil. Los hábitos alimentarios y de actividad física son relativamente saludables y similares en los niños con estado nutricional adecuado y con exceso de peso. No obstante, se reconocen algunas prácticas características de entornos obesogénicos, tales como el uso del televisor durante las comidas, la omisión del desayuno, el consumo de alimentos ricos en hidratos de carbono y el "picoteo" entre las comidas principales. Dichos hábitos conllevan a prevalencias más altas de exceso de peso infantil en San Carlos y en el casco urbano.

\section{Financiamiento}

Universidad Nacional de La Plata (11/N808), Agencia Nacional de Promoción Científica y Tecnológica (PICT-2016-0610) y Consejo Nacional de Investigaciones Científicas y Técnicas (CONICET, PIP 0106).

\section{Agradecimientos}

A los escolares, a sus padres y madres por su colaboración desinteresada. A las autoridades escolares y maestros que facilitaron nuestro trabajo de campo en los establecimientos educativos. A la Lic. Ivana Natalín Atadia y a nuestros compañeros del LINOA por la colaboración en el relevamiento y elaboración de la base de datos. A la Lic. María Antonia Luis y a la Sra. María Cristina Muñe por la revisión general del manuscrito. Al Arq. Luis María Forte por la asistencia gráfica. A las instituciones que brindaron su apoyo financiero.

\section{Biografía}

María Florencia Cesani. Licenciada en Antropología y doctora en Ciencias Naturales. Investigadora independiente del Consejo Nacional de Investigaciones Científicas Técnicas (CONICET) y jefa de Trabajos Prácticos de la Cátedra Antropología Biológica IV, Facultad de Ciencias Naturales y Museo, Universidad Nacional de La Plata (FCNyM, UNLP).

Evelia Edith Oyhenart. Licenciada en Antropología y doctora en Ciencias Naturales. Investigadora principal del Consejo Nacional de Investigaciones Científicas Técnicas (CONICET) y profesora titular de la Cátedra Antropología Biológica IV, Facultad de Ciencias Naturales y Museo, Universidad Nacional de La Plata (FCNyM, UNLP). 


\section{Q Referencias bibliográficas}

》 Abalde-Amoedo, N. y Pino-Juste, M. R. (2015). Influencia del entorno familiar y escolar en la práctica de actividad física. Revista de Estudios e Investigación en Psicología y Educación, 5, 112-116. doi: 10.17979/reipe.2015.0.05.363

»Aguirre, P. (2004). Ricos flacos y gordos pobres. La alimentación en crisis. Buenos Aires: Capital Intelectual. Colección Claves para Todos.

"Allén, A. (2003). La interfase periurbana como escenario de cambio y acción hacia la sustentabilidad del desarrollo. Cuadernos del CENDES, 53, 1-15.

" Alzugaray, L., Santa María, J. y Peiró, M. L. (2019). Cartografía del nivel socioeconómico de los hogares del Gran La Plata. Aplicación de un índice a partir de datos del Censo 2010. Ensenada: Universidad Nacional de La Plata, Facultad de Humanidades y Ciencias de la Educación, Instituto de Investigaciones en Humanidades y Ciencias Sociales (UNLP-CONICET). Documentos metodológicos IdIHCS. Cartografías, 2, 1-16. Recuperado de http://www.memoria.fahce.unlp.edu.ar/libros/pm.1070/pm.1070.pdf

» Bergel Sanchís, M. L., Cesani, M. F. y Oyhenart, E. E. (2017). Malnutrición infantil e inseguridad alimentaria como expresión de las condiciones socio-económicas familiares en Villaguay, Argentina (2010-2012). Un enfoque biocultural. Población y Salud en Mesoamérica, 14(2), 1-25. doi: 10.15517/psm.v14i2.27305

» Boswell, N., Byrne, R. y Davies, P. S. W. (2019). Family food environment factors associated with obesity outcomes in early childhood. BMC Obesity, 6, 17. doi: 10.1186/s40608019-0241-9

» Bustamante, M. J., Martínez, J. L., Alfaro, E. L., Sánchez Álvarez, M., Dipierri, J. E. y Tabera, C. (2019). Fracaso antropométrico y altura geográfica en escolares jujeños asistidos alimentariamente. Nutrición Clínica y Dietética Hospitalaria, 39(4), 16-23. doi: 10.12873/3943bustamante

"Carrasco Henríquez, N. (2007). Desarrollos de la antropología de la alimentación en América Latina: hacia el estudio de los problemas alimentarios contemporáneos. Estudios Sociales, 15(30), 80-101.

" Cordero, M. L. y Cesani, M. F. (2019). Nutritional transition in schoolchildren from Tucumán, Argentina: a cross-sectional analysis of nutritional status and body composition. American Journal of Human Biology, 31(4), e23257. doi: 10.1002/ajhb.23257

» de Onis, M., Onyango, A. W., Borghi, E., Siyam, A., Nishida, C. y Siekmann, J. (2007). Development of a WHO growth reference for school-aged children and adolescents. Bulletin of the World Health Organization, 85(9), 660-667. doi: 10.2471/BLT.07.043497

»Díaz Córdova, D. (2016). Novedades metodológicas aplicadas a la antropología alimentaria: modelos basados en agentes y redes sociales. Salud Colectiva, 12(4), 635-650. doi: 10.18294/sc.2016.1008

"Ferraro, R., Zulaica, L. y Echechuri, H. (2013). Perspectivas de abordaje y caracterización del periurbano de Mar del Plata, Argentina. Letras Verdes. Ecología Urbana. Programa de Estudios Socioambientales, 13, 19-40.

" Fischler, C. (2010). Gastro-nomía y gastro-anomía. Sabiduría del cuerpo y crisis biocultural de la alimentación moderna. Gazeta de Antropología, 26(1), 09. Recuperado de http://hdl.handle.net/10481/6789 
»Franco Patiño, S. M. (2010). Aportes de la sociología al estudio de la alimentación familiar. Revista Luna Azul, 31, 139-155.

»Frediani, J. C. (2009). Las nuevas periferias en el proceso de expansión urbana. El caso del partido de La Plata. Geograficando: Revista de Estudios Geográficos, 5(5), 103-125. Recuperado de http://geograficando.fahce.unlp.edu.ar

»Frediani, J. C. (2010). Lógicas y tendencias de la expansión residencial en áreas periurbanas. El Partido de La Plata, Buenos Aires, Argentina, entre 1990 y 2010. (tesis de doctorado), Universidad Nacional de La Plata, Facultad de Humanidades y Ciencias de la Educación, La Plata, Argentina. Recuperado de http://sedici.unlp.edu.ar/handle/10915/3203

» García Barthe, M. (2014). Del alimento a la comida. Las transformaciones en la comensalidad y su efecto en los cuerpos de los niños. Revista del Hospital de Niños (Buenos Aires), 56(255), 237-248.

» Garraza, M., Cesani, M. F., Navone, G. T. y Oyhenart, E. E. (2016). Malnutrition and body composition in urban and rural schoolchildren: a cross-sectional study in San Rafael, Mendoza (Argentina). American Journal of Human Biology, 28(6), 796-803. doi: 10.1002/ajhb.22869

»Ihmels, M. A., Welk, G. J., Eisenmann, J. C. y Nusser, S. M. (2009). Development and preliminary validation of a Family Nutrition and Physical Activity (FNPA) screening tool. International Journal of Behavioral Nutrition and Physical Activity, 6, 14. doi: 10.1186/14795868-6-14

» Johnson, R., Welk, G., Saint-Maurice, P. F. e Imhels, M. (2012). Parenting styles and home obesogenic environments. International Journal of Environmental Research and Public Health, 9(4), 1411-1426. doi: 10.3390/ijerph9041411

» Koh, G. A., Scott, J. A., Woodman, R. J., Kim, S. W., Daniels, L. A. y Magarey, A. M. (2014). Maternal feeding self-efficacy and fruit and vegetable intakes in infants. Results from the SAIDI study. Appetite, 81, 44-51. doi: 10.1016/j.appet.2014.06.008

» Lázaro Cuesta, L., Rearte, A., Rodríguez, S., Niglia, M., Scipioni, H., Rodríguez, D., Salinas, R., Sosa, C. y Rasse, S. (2018). Estado nutricional antropométrico, bioquímico e ingesta alimentaria en niños escolares de 6 a 14 años, General Pueyrredón, Buenos Aires, Argentina. Archivos Argentinos de Pediatría, 116(1), e34-e46. doi: 10.5546/aap.2018.e34

» Lohman, T. G., Roche, A. F. y Martorell, R. (1988). Anthropometric standardization reference manual. Champaign, IL: Human Kinetics Books.

» Macias, A. I. M., Gordillo, L. G. S. y Camacho, E. J. R. (2012). Hábitos alimentarios de niños en edad escolar y el papel de la educación para la salud. Revista Chilena de Nutrición, 39(3), 40-43. doi: 10.4067/So717-75182012000300006

» Ministerio de Salud de la Nación. (2013). Sobrepeso y obesidad en niños y adolescentes. Orientaciones para su prevención, diagnóstico y tratamiento en Atención Primaria de la Salud. Buenos Aires: Ministerio de Salud de la Nación.RR

" Ministerio de Salud y Desarrollo Social. (2019). 2ํㅡㄹ Encuesta Nacional de Nutrición y Salud (ENNyS 2). Indicadores Priorizados. Buenos Aires, Argentina: Ministerio de Salud y Desarrollo Social. Recuperado de https://bancos.salud.gob.ar/recurso/2deg-encuestanacional-de-nutricion-y-salud-indicadores-priorizados

» Moore, L. L., Lombardi, D. A., White, M. J., Campbell, J. L., Oliveira, S. A. y Ellison, C. (1991). Influence of parents " physical activity levels on activity levels of young children. The Journal of Pediatrics, 118(2), 215-219. doi: 10.1016/Soo22-3476(05)80485-8

» Moscoso Sánchez, D., Sánchez García, R., Martín Rodríguez, M. y Pedrajas Sanz, N. (2015). ¿Qué significa ser activo en una sociedad sedentaria? Paradojas de los estilos de vida y el ocio en la juventud española. EMPIRIA. Revista de Metodología de Ciencias Sociales, 30, 77-108. doi: empiria.30.2015.13886 
» Navazo, B., Oyhenart, E. E. y Dahinten, S. L. (2019). Doble carga de malnutrición y nivel de bienestar socio-ambiental de escolares de la Patagonia argentina (Puerto Madryn, Chubut). Nutrición Clínica y Dietética Hospitalaria, 39(2), 111-119. doi: 10.12873/392navazo

» Organización Mundial de la Salud. (2020). Malnutrición. Recuperado de https://www. who.int/es/news-room/fact-sheets/detail/malnutrition

» Oyhenart, E. E., Castro, L. E., Forte, L. M., Sicre, M. L., Quintero, F. A., Luis, M. A., Torres, M. F., Luna, M. E., Cesani, M. F. y Orden, A. B. (2008). Socioenvironmental conditions and nutritional status in urban and rural schoolchildren. American Journal of Human Biology, 20(4), 399-405. doi: 10.1002/ajhb.20738

" Oyhenart, E. E., Torres, M. F., Luis, M. A., Luna, M. E., Castro, L. E., Garraza, M., Navazo, B., Fucini, M. C., Quintero, F. A. y Cesani, M. F. (2018). Estudio comparativo del estado nutricional de niños y niñas residentes en cuatro partidos de la provincia de Buenos Aires (Argentina), en el marco de la transición nutricional. Salud Colectiva, 14(3), 597-606. doi: $10.18294 / \mathrm{sc} .2018 .1576$

» Pellegrini, M. y Battistini, N. C. (2015) Nutritional status evaluation: body composition and energy balance. En A. Lenzi, S. Migliaccio y L.M. Donini (Eds.), Multidisciplinary Approach to Obesity (pp. 171-192). Cham: Springer.

" Pocock, M., Trivedi, D., Wills, W., Bunn, F. y Magnusson, J. (2009). Parental perceptions regarding healthy behaviours for preventing overweight and obesity in young children: a systematic review of qualitative studies. Obesity reviews, 11(5), 338-353. doi: 10.1111/j.1467789X.2009.00648.X

" Rodríguez Tarducci, R. (2018). Asentamientos informales en el partido de La Plata. Una aproximación a las modalidades de ocupación del territorio. Estudios Socioterritoriales. Revista de Geografía, 23, 119-136.

" Spurrier, N. J., Magarey, A. A., Golley, R., Curnow, F. y Sawyer, M. G. (2008). Relationships between the home environment and physical activity and dietary patterns of preschool children: a cross-sectional study. International Journal of Behavioral Nutrition and Physical Activity, 5, 31. doi: 10.1186/1479-5868-5-31

» Swinburn, B., Egger, G. y Raza, F. (1999). Dissecting obesogenic environments: the development and application of a framework for identifying and prioritizing environmental interventions for obesity. Preventive Medicine, 29, 563-570.

» Thompson, N., Mansfield, B., Stringer, M., Stewart, B., Potter, J. y Fernengel, K. (2016). An evidence-based resource for the management of comorbidities associated with childhood overweight and obesity. Journal of the American Association of Nurse Practitioners, 28(10), 559-570. doi: 10.1002/2327-6924.12369

» Torres, M. F. (2012). Malnutrición y heterogeneidad socio-ambiental. Un análisis en escolares urbanos de 9 a 16 años residentes en La Plata, Argentina. Runa, archivo para las ciencias del hombre, 33(1), 85-106. doi: 10.34096/runa.v33i1.341

》UNICEF. (2019). The State of the World's Children 2019. Children, food and nutrition: growing well in a changing world. Nueva York: UNICEF.

"Wanderley, E. N. y Ferreira, V. A. (2010). Obesidade: uma perspectiva plural. Ciência e Saúde Coletiva, 15(1), 185-194. doi: 10.1590/S1413-81232010000100024

»Wijtzes, A. I., Jansen, W., Bouthoorn, S. H., van Lenthe, F. J., Franco, O. H., Hofman, A., Jaddoe, V. W. V. y Raat, H. (2016). Meal-skipping behaviors and body fat in 6-year-old children. The Journal of Pediatrics, 168, 118-125.e2. doi: 10.1016/j.jpeds.2015.09.039

"World Health Organization. (2017). Report of the Commission on Ending Childhood Obesity. Geneva: World Health Organization. Recuperado de: https://apps.who.int/iris/ bitstream/handle/10665/259349/WHO-NMH-PND-ECHO-17.1-eng.pdf 
